\title{
OPTIMIZED ZONAL STABLE ELECTION PROTOCOL
}

\author{
Ashwin S, Bharanidharan A, Harish A, Mohamed Afreez M \\ Department of Electronics and Communication Engineering \\ SRM Institute of Science and Technology, Kancheepuram, Tamil Nadu, India
}

\begin{abstract}
Group of sensor nodes that are dedicated to perform sensing task is called Wireless Sensor Networks (WSN). Clustering is implemented to make WSN process for Precision Agriculture (PA), energy efficient. A routing protocol is what makes the process efficient. We have proposed a routing protocol with sleep/wake scheduling and VANET broadcasting. Our aim is to implement a protocol and to prove that it is efficient (in terms of energy consumption and throughput) on comparison and analysis with the existing protocols such as Z-Stable Election Protocol and Stable Election Protocol, is what we are working for.
\end{abstract}

\section{Keywords - VANET, sleep/wake, network lifetime}

\section{INTRODUCTION}

In recent years, there has been a decrease in production from agricultural industry relative to the growing population in the country. Also, there has been an enormous development in the wireless sensor technologies. Use of WSN in the field has proved to yield better efficiency in meeting agricultural and consumer demands. Among many different types of PA, WSN is becoming commercial. Implementation of PA helps to monitor various parameters in the field and act as programmed. Various parameters that could be measured by using WSN for PA are soil, water, temperature and also help in disease monitoring. PA takes four different perspectives into account: Agronomical, Environmental, Technical, and Economical. We have proposed a protocol that aims to increase the energy efficiency of WSN. The protocol we have proposed was obtained by modifying the already existing protocol's architecture.

The rest of the paper is organized as follows. Proposed ns2 algorithm and system design are detailed in section II. Simulation and performance analysis are presented in section III. Conclusion is presented in section IV.

\section{PROPOSED ALGORITHM}

The efficiency of WSN can be increased in two ways: 1) Energy Harvesting Technique, 2) Energy consumption technique. We have chosen to achieve our objective by employing a method/algorithm that concentrates of energy consumption of WSN. The parameters that decide the efficiency are 1) Network Lifetime, 2) Energy Consumption, 3) Throughput.

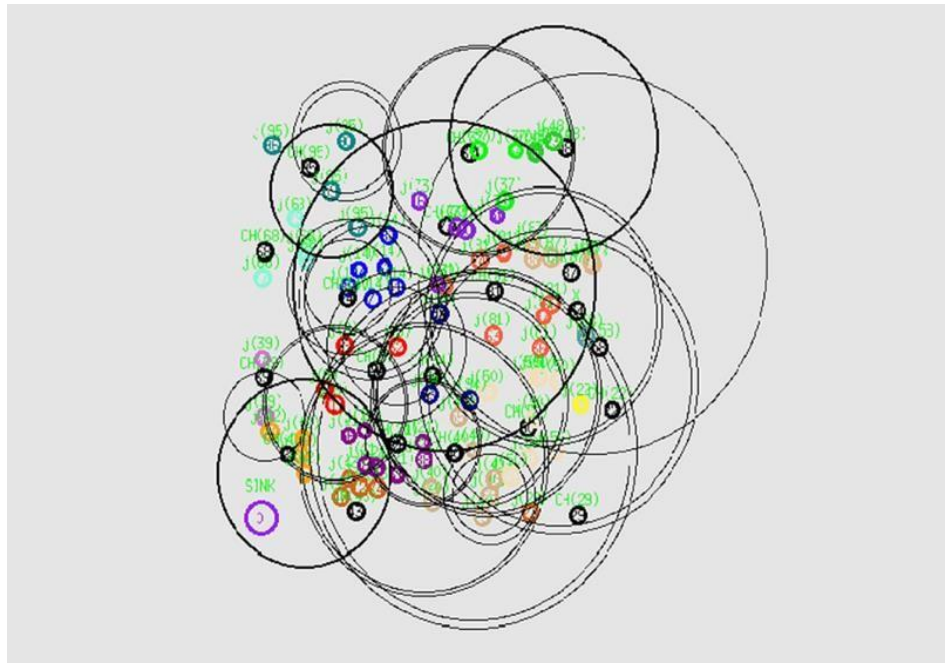

Fig. 1. WSN Implementation

The sleep-awake scheduling technique employed in the proposed system helps in in- creasing network lifetime and decreasing energy consumption VANET increases packet throughput. The nodes are deployed randomly in the field. Node 0 is the sink node. The nodes sense the data and broadcast the message. Calculation of energy level of nodes takes place. The Cluster Head election is done after the formation of cluster. $\mathrm{CH}$ selection process is based on optimum probability election method, which is dependent on the energy levels of the nodes. The $\mathrm{CHs}$ are the ones that directly communicate with the Sink node. CHs collects data only up to certain distance called the threshold distance. The sensed data is sent to the sink node only if data is above a particular threshold level. The packets are used as messages for communication; UDP is employed. Since, the nodes are not routers we have used CBR as the traffic source using UDP as traffic agent. The VANET is used as the nodes are not stationery. 


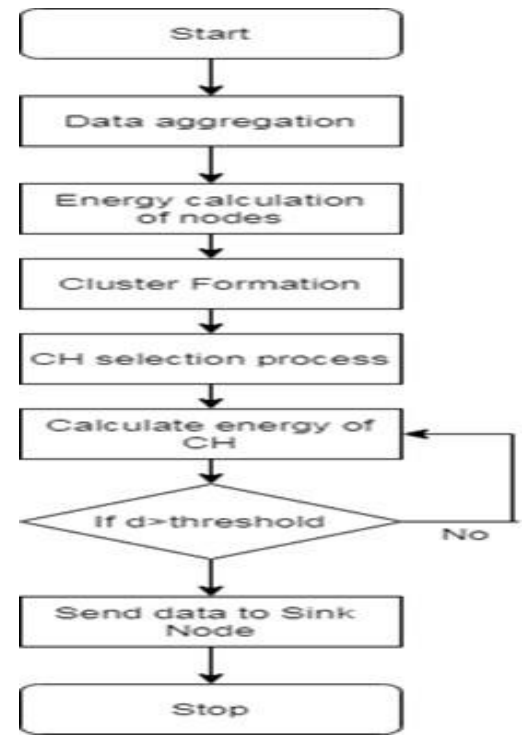

Fig. 2. System Design

In general sleep/wake scheduling protocol is used for limiting start to finish delay for occasion driven multi-bounce remote sensor systems. Rest/wake planning plan is subject to traffic loads. Hubs adjust to rest/wake plan dependent on traffic stacks by virtue of three significant variables, (a) the separation of the hub from the sink hub, (b) the significance of the hub's area from network's point of view, and (c) if the hub is in the vicinity where an occasion happens. Utilizing these heuristics, there is a decreased start to finish delay and sensibly great increment in the throughput by smaller than usual mizing the clog at hubs that have overwhelming traffic load. Rest/wake strategy has been proficient in diminishing the start to finish delay, just as has improved the different QoS parameters, similar to average postponement, parcel misfortune proportion, and throughput in various protocols.

The proposed protocol uses VANET broadcasting technique. The messages i.e., the packets that are transmitted from the nodes have to follow a set of rules and these are laid by the above shown code. The parameters decide how far the packets can be broadcasted by a node and in the specified interval. This type of ad hoc network plays an important role in congestion and traffic monitoring also ensures the safety of packages. VANETs provide better mobility, they are robust, spontaneous also flexible. awk script is used to process the trace file and produce the throughput result. The system can be used in PA to measure different soil parameters for enhancing the quantity and quality of crops. The proposed protocol can be used to gather and process large amount of data from beginning of monitoring and manage air quality, the conditions of traffic, to weather situations. Also, it is adaptable to different wireless technologies. The ns2 was used to show the implementation of WSN and to display each of the nodes behavior over time. MATLAB software was used for comparison and analysis of the proposed protocol with preexisting protocols. We have used UDP as the traffic agent whose source (traffic source) is CBR. MAC layer follows IEEE standard - IEEE 802.11.

\section{EXPERIMENT AND RESULT}

We have used 150x300 as the dimensional metric of the field for the simulation part of the proposed protocol. The nodes change their colors during different stages of their rounds. The different stages in WSN being 1) Energy Calculation, 2) Cluster formation, 3) $\mathrm{CH}$ selection process, 4) Nodes send the join request to $\mathrm{CH}$ to become a cluster member,

5) $\mathrm{CHs}$ accepting the request and the node will be included as its cluster member, 6) The stage where the message is transferred from cluster members to $\mathrm{CHs}$, then $\mathrm{CHs}$ receive and aggregate the messages from different nodes and sends them to the Sink node.

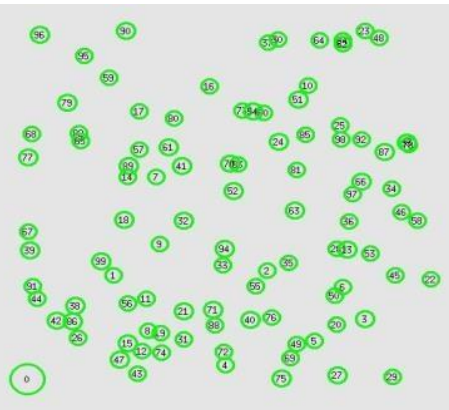

(a)

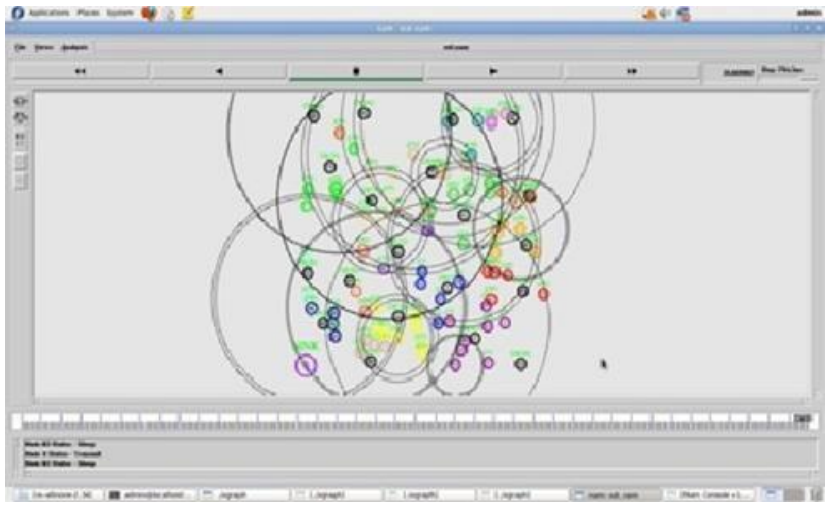

(b)

Fig. 3. (a) Node arrangement (b) Behaviour of WSN after few rounds.

The different stages can be made to happen at the custom time interval and we have chosen the time difference to be as five seconds between each stage. Each node is supposed to have a threshold distance, the distance up to which their range exists. That range is the maximum amount of field the nodes can broadcast their messages. The threshold distance is calculated based on a formula. The formula is based on the XY co- ordinates of a node in the field. The nodes are assigned energy using uniform random variable function within the limits of minimum energy $(0.9 \mathrm{~J})$ and maximum energy (1.1J). 
International Journal of Engineering Applied Sciences and Technology, 2020

Vol. 5, Issue 1, ISSN No. 2455-2143, Pages 413-416

Published Online May 2020 in IJEAST (http://www.ijeast.com)

\begin{tabular}{|l|c}
\hline \multicolumn{1}{|c|}{ Parameters Used } & Values \\
\hline Size of the network field & $150 \mathrm{~m}, 300 \mathrm{~m}$ \\
\hline Number of noces & 100 \\
\hline MAC & $\mathbb{E E E ~ 8 0 2 . 1 1 ~}$ \\
\hline Maximum packet length in quelle & 50 \\
\hline Receiver threshold & $.88 \mathrm{dbm}$ \\
\hline Transmission threshold & $.85 \mathrm{dbm}$ \\
\hline Packet Size & 512 \\
\hline
\end{tabular}

Table 1: Values used for during simulation.

minimum energy (0.9J) and maximum energy (1.1J). The Network lifetime is the analysis of number of nodes that are alive against the number of rounds. A WSN is said to have better network lifetime depending on how long the nodes can withstand without draining the energy completely. A node loses en- ergy in two ways; 1) Sensing of the information it is designated for, 2) Transmission of the sensed information. In case of $\mathrm{CH}$, the energy usage depends on transmission and reception of messages to/from the nodes and communication between the sink nodes. The MATLAB simulation for comparison between SEP, ZSEP, and OZSEP (pro- posed protocol) is done by taking the number of advanced nodes which is represented by the variable $m$ that is equal to 0.1 and the energy of advanced nodes (a) equal to 2 .

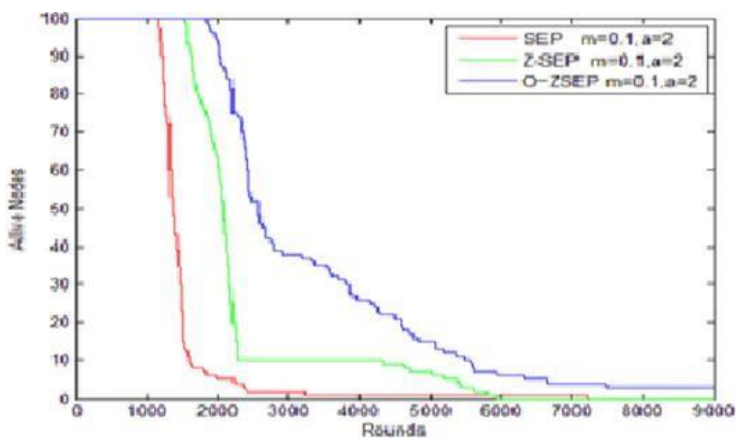

(a) Network lifetime

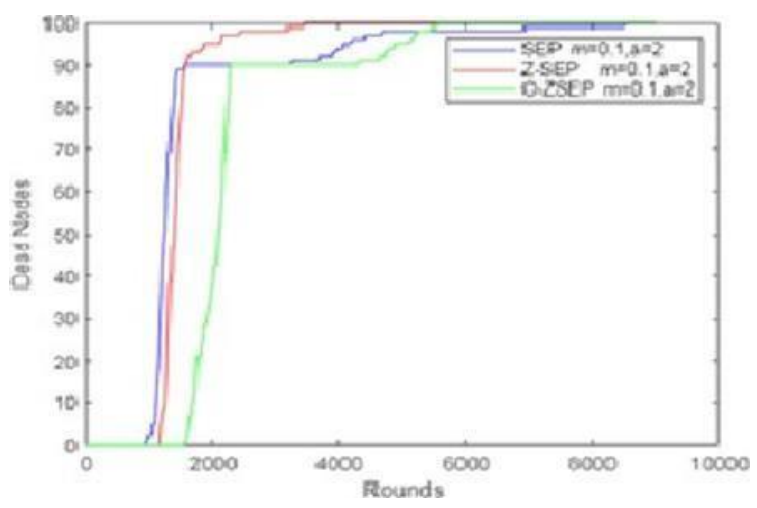

(b) Energy Consumption

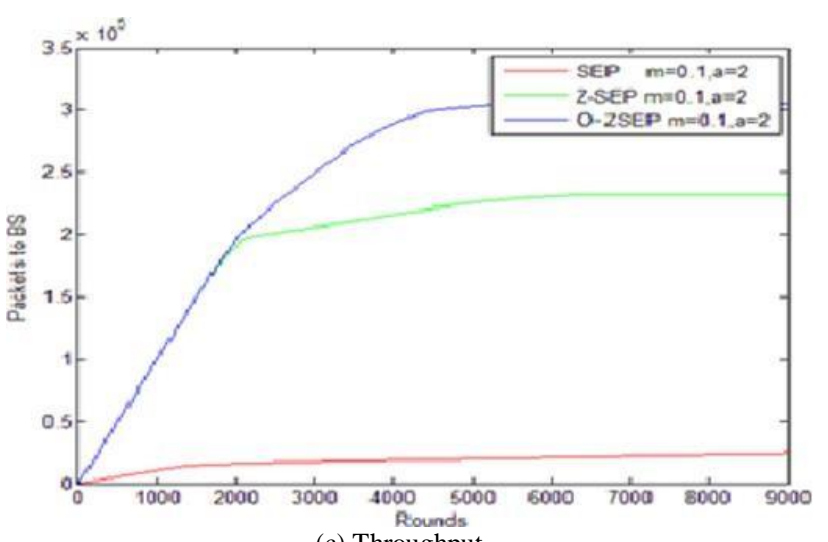

(c) Throughput

Fig. 4. (a) Network Lifetime (b) Energy Consumption (c) Throughput

Table -2 Experiment Result

\begin{tabular}{|c|c|c|c|}
\hline Protocol & $\begin{array}{c}\text { Lifetime } \\
\text { (rounds) }\end{array}$ & $\begin{array}{c}\text { Stability } \\
\text { period } \\
\text { (rounds) }\end{array}$ & $\begin{array}{c}\text { Throughput } \\
\text { (rounds) }\end{array}$ \\
\hline SEP & 3200 & 1400 & $3.7 \times 10^{4}$ \\
\hline ZSEP & 5900 & 1550 & $1.7 \times 10^{5}$ \\
\hline OZSEP & 7400 & 1820 & $2.1 \times 10^{5}$ \\
\hline
\end{tabular}

OZSEP outperforms the other two protocols in the network lifetime analysis due to the clustering technique and sleep/wake scheduling. This also shows that OZSEP prolonged the stability period. The above image shows us that nodes die at 3200, 5900, and 7400 in SEP, ZSEP and OZSEP respectively. Also, the proposed protocol performs better in the energy consumption which is a metric for number of dead nodes against the number of rounds. Throughput is defined as the numerical of total number of packets sent to the base station against the number of rounds. Throughput is a measure for packet delivery ratio that is the number of packets acquired by the sink nodes, which were sent from the sensing nodes. From the table and MATLAB plots, it is evident that the proposed protocol has more energy efficiency i.e., the energy consumption is lesser (it can be seen by increase in stability period), network lifetime is more and Packet delivery ratio is more (as evident from throughput data).

\section{CONCLUSION}

In this project, we have proposed an algorithm (OZSEP) through a new arrangement of nodes and selection of cluster heads in order to improve the energy efficiency of wireless sensor nodes. The OZSEP (Optimized zonal stable election protocol) shows enhanced performance in network lifetime, throughput and decreased energy consumption. The simulation results show that the energy efficiency of OZSEP is better than the Zonal Stable Election Protocol and Stable Election Protocol at about 12.8 percent and

19.5 percent respectively, through large topology heterogeneous network (100 nodes and $150 \mathrm{~m} \times 300 \mathrm{~m})$. The proposed approach will be increasingly significant when the remote sensor organize is conveyed in huge region and the BS 
is a long way from the system. In future the system lifetime, solidness and throughput can be additionally improved by changing the sink portability as per the $\mathrm{CHs}$ area and introduction of data security to the protocol will help in reallife implementation.

\section{REFERENCE}

[1] Jagadeesh Naik L.; Ramanaiah K.V.; Soundara Rajan K. (2019). "Performance Evaluation of MCHSEP and SEP protocol for Wireless Sensor Networks"; (IJRTE) ISSN: 2277-3878, Volume-7, Issue-6S4 (pp.2277-3878).

[2] Sulekha Kumari, Jeetu Sharma, Partha Prathim Bhattachriya (2017); "Performance Evaluation of SEP, LEACH and ZSEP under the Influence of Network Area"; (pp.85-91).

[3] Khriji, Kamel Besbes, Ines Kammoun, Dhouha El Houssani (2019); "Energy Efficient Routing algorithm based on localization and clustering techniques for agricultural applications" IEEE, AE Systems Magazine, (pp 56-66).

[4] Dr. Deepti Gupta (2015); "Wireless Sensor Networks "Future trends and Latest Research Challenges"'; IOSR Journal of Electronics and Communication Engineering (IOSR-JECE) Volume 10, Issue 2, Ver. II. (pp.41-46).

[5] Asmita Singh, Abhishek Tyagi, Dr Sindhu Hak (2019); "Energy Efficient WSN for Precision Agriculture -Using Modified Zonal Stable Election Protocol"; (DOI: 10.1109/SPIN.2019.8711694).

[6] Sidiq Iqbal, Sandeep Gowda, Mahesh B.S. (2014); "Performance Analysis of Stable Election Protocol and its Extensions in WSN"; (DOI: 10.1109/ICACCCT.2014.7019189).

[7] Muhammad Rizwan Ghori, Kamal Z. Zamli, Nik Quosthoni, Muhammad Hisyam, Mohamed Montaser (2018); "Vehicular Ad-hoc Network (VANET): Review"; 2018 IEEE International Conference on Innovative Research and Development DOI: 10.1109/ICIRD.2018.8376311 .

[8] Venkateswarlu Kumaramangalam M, Adiyapatham K, Kandasamy C,(2014). "Zone- based routing protocol for wireless sensor networks". Int. Sch. Res. Not. Vol. 2014, article ID 798934. (DOI: 10.1155/2014/798934).

[9] Kashaf A; Javaid N; Khan Z.A; Khan I.A (2012); “ TSEP: Threshold Sensitive Stable Election Protocol for WSNs"; 2012 10th International Conference on Frontiers of Information Technology (DOI: 10.1109/FIT.2012.37).

[10] https://www.geeksforgeeks.org/basics-of-ns2-and-otcltclscript/ , "Basics of ns2".

[11] http://www.mathcs.emory.edu/cheung/Courses/558/Sylla bus/18-WirelessSim/intro.html, "Declaration, Definition and Configuration of WSN".

[12] https://slogix.in/ "Sample ns2 source codes".

[13] https://en.wikipedia.org/wiki/Wirelesssensornetwork Overviewof WSN. 